\title{
HSC/ 14/ 04
}

\section{A note on using the Hodrick-Prescott filter in electricity markets}

\author{
Rafał Weron ${ }^{1}$ \\ Michał Zator ${ }^{1}$
}

${ }^{1}$ Institute of Organization and Management, Wrocław University of Technology, Poland

Hugo Steinhaus Center

Wrocław University of Technology

Wyb. Wyspiańskiego 27, 50-370 Wrocław, Poland http://www.im. pwr.wroc. pl/ hugo/ 


\title{
A note on using the Hodrick-Prescott filter in electricity markets
}

\author{
Rafał Weron ${ }^{\mathrm{a}}$, Michał Zator ${ }^{\mathrm{a}}$ \\ ${ }^{a}$ Institute of Organization and Management, Wrocław University of Technology, 50-370 Wroctaw, Poland
}

\begin{abstract}
Recently, Nowotarski et al. (2013) have found that wavelet-based models for the long-term seasonal component (LTSC) are not only better in extracting the LTSC from a series of spot electricity prices but also significantly more accurate in terms of forecasting these prices up to a year ahead than the commonly used monthly dummies and sine-based models. However, a clear disadvantage of the wavelet-based approach is the increased complexity of the technique as compared to the other two classes of LTSC models, which may render it too complicated for practitioners. To facilitate this problem, we propose here a much simpler, yet equally powerful method for identifying the LTSC in electricity spot price series. It makes use of the Hodrick-Prescott (HP) filter, a widely-recognized tool in macroeconomics.
\end{abstract}

Keywords: Hodrick-Prescott filter, Electricity spot price, Long-term seasonal component, Robust modeling

\section{Introduction}

A fundamental issue in the process of building stochastic models for electricity spot prices is the treatment of trends and seasonality in the data (Bierbrauer et al., 2007; Janczura et al., 2013). It is well known that no matter from which market it originates, the electricity spot price exhibits seasonality on the daily, weekly and - to some extent - on the annual level. While for short-term horizons of a few minutes to a few days ahead the latter may be ignored and the focus is on the daily and weekly seasonalities, in the mid-term (a few days up to a few months ahead) the daily profile becomes irrelevant as most models work with average daily prices, but the long-term seasonal component (LTSC) plays a crucial role. Its misspecification can introduce bias or artificial price variability. This may result in a bad estimate of the mean reversion level or of the price spike intensity and severity; consequently, in underestimating risk and even in incurring financial losses (Trück et al., 2007; Weron, 2014).

The annual seasonality is present in electricity spot prices due to changing weather conditions throughout the year. However, in most markets it is dominated by a more irregular cyclic component, dependent on macroeconomic variables (like fuel prices and economic growth) and long-term weather trends (e.g. lower than historical precipitation or temperatures). In the time series literature this would be called a trend-cycle component, in electricity price modeling it is

Email addresses: rafal.weron@pwr.wroc.pl (Rafał Weron), michal.zator@pwr.wroc.pl (Michał Zator) 
rather referred to as a trend-seasonal or seasonal component, to reflect the underlying annual seasonality. In the energy economics literature there are essentially three approaches to modeling the LTSC:

- piecewise constant functions or dummies, possibly combined with a linear trend (see e.g. Fanone et al., 2013; Fleten et al., 2011; Gianfreda and Grossi, 2012; Haugom and Ullrich, 2012; Higgs and Worthington, 2008),

- sinusoidal functions or sums of sinusoidal functions of different frequencies (see e.g. Benth et al., 2012; Bierbrauer et al., 2007; De Jong, 2006; Keles et al., 2012; Weron, 2008),

- wavelets (see e.g. Conejo et al., 2005; Janczura and Weron, 2010, 2012; Schlueter, 2010; Stevenson et al., 2006; Weron, 2006) or other nonparametric smoothing techniques (like Friedman's supersmoother, see Bordignon et al., 2013).

In an extensive study on the estimation and forecasting of the LTSC, Nowotarski et al. (2013) considered a battery of over 300 models from the above three classes. They found that the waveletbased models were not only better in extracting the LTSC from a series of spot electricity prices but also significantly better in terms of forecasting these prices up to a year ahead than the commonly used monthly dummies and sine-based models. However, a clear disadvantage of the waveletbased approach - not mentioned in the cited paper - is the increased complexity of the technique as compared to the other two classes of LTSC models. The lack of ready-to-use software codes for computing the wavelet transform in a number of programming and forecasting environments may render the wavelet-based approach too complicated for practitioners.

To facilitate this problem, we propose here a much simpler, yet equally powerful method for identifying the LTSC in electricity spot price series. It makes use of the Hodrick-Prescott (HP) filter, a widely-recognized tool in macroeconomics. Although the filter was initially designed for a somewhat different purpose, it proves to work quite well in the context of electricity markets. In Section 2 we describe the method, then in Section 3 we perform an empirical exercise to show the effectiveness of the HP filter in extracting the LTSC of electricity spot prices. Finally, in Section 4 we conclude.

\section{The Hodrick-Prescott filter and electricity spot prices}

The approach we advocate here is based on the so-called Hodrick and Prescott $(1980,1997)$ filter. The HP filter was originally intended for decomposing the series of GDP values into a long-term growth component and a cyclical component. The mechanics of the filter are, however, universal - split the series into two parts, a smooth and a volatile one. In macroeconomics the smooth part corresponds to long-term growth and the volatile part to the business cycle. In the context of electricity markets we may interpret the smooth part as the long-term (trend-)seasonal component and the volatile part as the stochastic component.

The filter is a non-parametric method, which returns a smoothed series $\tau_{t}$ for a noisy (or volatile) input series $y_{t}$. Values $\tau_{t}$ are chosen as to solve the following minimization problem:

$$
\min _{\tau_{t}}\left\{\sum_{t=1}^{T}\left(y_{t}-\tau_{t}\right)^{2}+\lambda \sum_{t=2}^{T-1}\left[\left(\tau_{t+1}-\tau_{t}\right)-\left(\tau_{t}-\tau_{t-1}\right)\right]^{2}\right\} .
$$




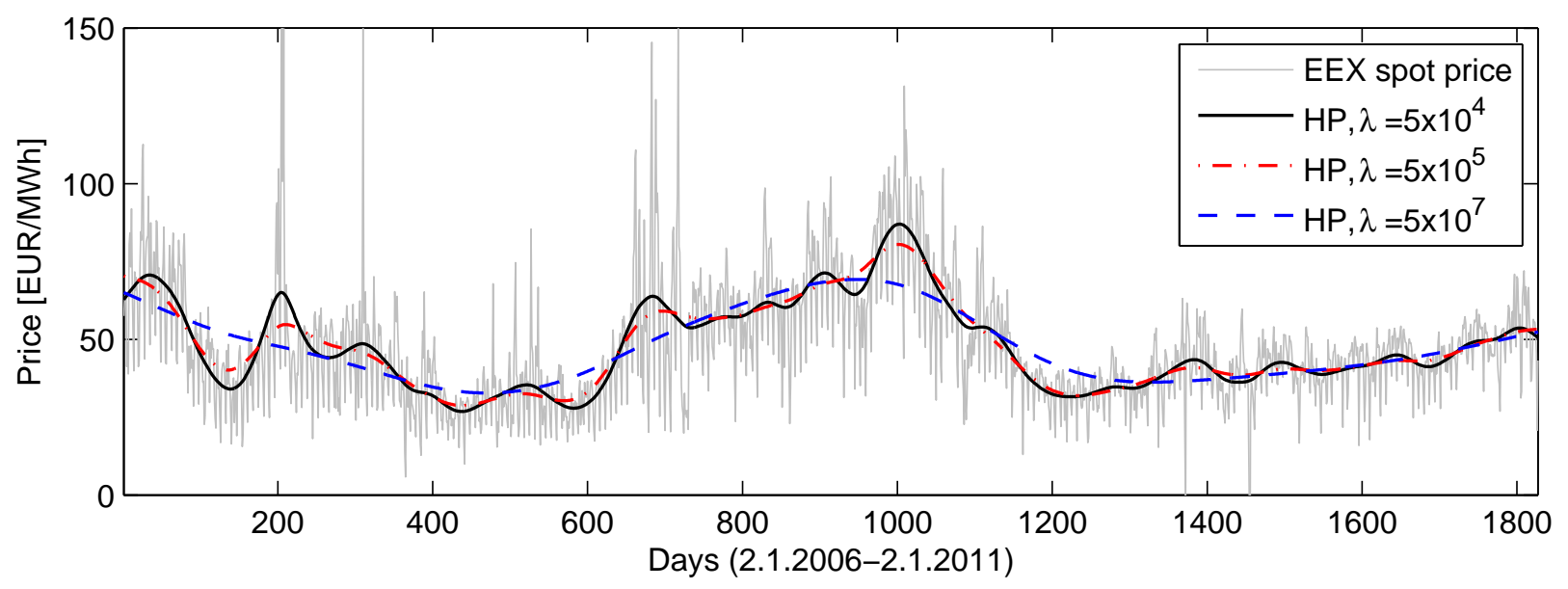

Figure 1: Five years (2.1.2006-2.1.2011) of daily electricity spot prices from the European Energy Exchange (EEX, Germany) together with three sample smoothed series obtained by applying the HP filter with different values of smoothing parameter $\lambda$.

The first sum is the punishment for deviating from the original series and the second is the punishment for roughness of the smoothed series. The larger the value of $\lambda$, the higher is the latter penalty. For technical issues behind the method we refer to the original paper of Hodrick and Prescott (1997) and the quite rich literature devoted to the filter. The filter is readily available in many econometric and statistical software environments. For instance, in Matlab the function to compute the HP filter (hpfilter.m) is part of the Econometric Toolbox. It is also straightforward to implement it in most programming languages.

\section{Empirical evidence}

Let us now investigate the performance of the HP filter in the context of electricity markets. We first illustrate the effects of applying the filter for different values of smoothing parameter $\lambda$. In Figure 1 we plot the original price series from the European Energy Exchange (EEX, Germany) together with three sample fits of the Hodrick-Prescott filter for different values of $\lambda$. Apparently, the HP filter can approximate the trend-seasonal pattern as closely as needed. We must therefore find a way to choose a proper smoothing parameter; note that an analogous problem is faced when choosing the level of the wavelet decomposition, see e.g. Nowotarski et al. (2013). Hodrick and Prescott (1997) provide a rough estimate of the optimal smoothing parameter and argue that the results are not very sensitive to the parameter's value. However, they work with quarterly and annual macroeconomic data and the value of $\lambda$ for higher-frequency data has to be chosen differently.

To find which $\lambda$ 's perform best for datasets typically encountered in mid-term modeling, i.e. for lead times measured in months, we apply the HP filter to 3- to 8-year long daily spot price series from three markets (EEX, Nord Pool and PJM) and compare the fits with those obtained by applying two state-of-the-art LTSC models:

- A wavelet smoother based on the Daubechies wavelet family of order 24 and 5, 6, 7 or 8 
levels of decomposition, i.e. $S_{5}, S_{6}, S_{7}$ and $S_{8}$ approximations, respectively; for details see Janczura et al. (2013) or Nowotarski et al. (2013).

- A sine of a 365 day period combined with an exponentially weighted moving average and smoothing parameter 0.975 ('sin-EWMA'; as suggested by De Jong, 2006). For implementation details see also Janczura et al. (2013).

We find that the value of $\lambda$ that minimizes squared differences between the HP filter LTSC and the wavelet or sin-EWMA LTSC varies from roughly $5 \cdot 10^{4}$ to roughly $5 \cdot 10^{7}$, depending on the smoothness of the latter. In the second part of the empirical exercise we therefore decide to use seven different $\lambda$ 's spanning this range: $5 \cdot 10^{4}, 1 \cdot 10^{5}, 5 \cdot 10^{5}, 1 \cdot 10^{6}, 5 \cdot 10^{6}, 1 \cdot 10^{7}$ and $5 \cdot 10^{7}$.

We now check whether the HP filter is able to correctly identify the seasonal pattern. Obviously, we do not know the true seasonal pattern and hence we take an approach similar to that of Janczura et al. (2013). Namely, we apply the following simulation procedure:

1. Estimate the LTSC of a given empirical series of spot prices using a chosen technique (called the input technique in Table 1) and divide the series into stochastic and trend-seasonal components.

2. Using the stochastic component identified in step 1, estimate the parameters of a three state Markov-regime switching (MRS) model, see Janczura and Weron (2010) or Nowotarski et al. (2013) for details.

3. Simulate $N=1000$ trajectories of the stochastic component estimated in step 2 and add them to the LTSC estimated in step 1, to obtain 1000 artificial (i.e. simulated) trajectories resembling the spot price series.

4. Use a HP filter with a chosen $\lambda$ (called the identification technique in Table 1) to identify the seasonal pattern in the artificial trajectories generated in step 3.

5. Compute the squared differences (i.e. mean squared errors, MSE) between the estimated seasonal pattern from step 4 and the original, seasonal pattern identified in step 1.

We repeat this exercise for three markets (Nord Pool, EEX and PJM; see also Figures 1 and 2) using different input techniques (wavelets with 5, 6, 7 and 8 levels of decomposition and a combination of sines and exponential smoothing) as well as different identification techniques (seven HP filters with $\lambda$ ranging from $5 \cdot 10^{4}$ to $5 \cdot 10^{7}$ and the four original input techniques). A sample simulated trajectory from step 3 together with the 'true' $S_{6}$ wavelet-based LTSC and the HP filter-based LTSC for $\lambda=5 \cdot 10^{5}$ is presented in Figure 2. We compare the average squared differences between the HP-based and the 'original' LTSC (see step 1 above) in Table 1. Note that not always the input technique turns out to be the best identification technique. This is especially true for the poorly performing sin-EWMA LTSC.

As we can see, the Hodrick-Prescott filter proves to be a good method of extracting the longterm (trend-)seasonal component from the data. For all analyzed markets and input techniques the best fit is obtained either by the input technique itself or by one of the HP filters. There seems to be a relatively universal relation between the optimal smoothing parameter $(\lambda)$ and the level $(k)$ of the wavelet decomposition used as the input technique: a HP filter with $\lambda \approx 5 \cdot 10^{k-1}$ roughly corresponds to the $S_{k}$ wavelet approximation. 


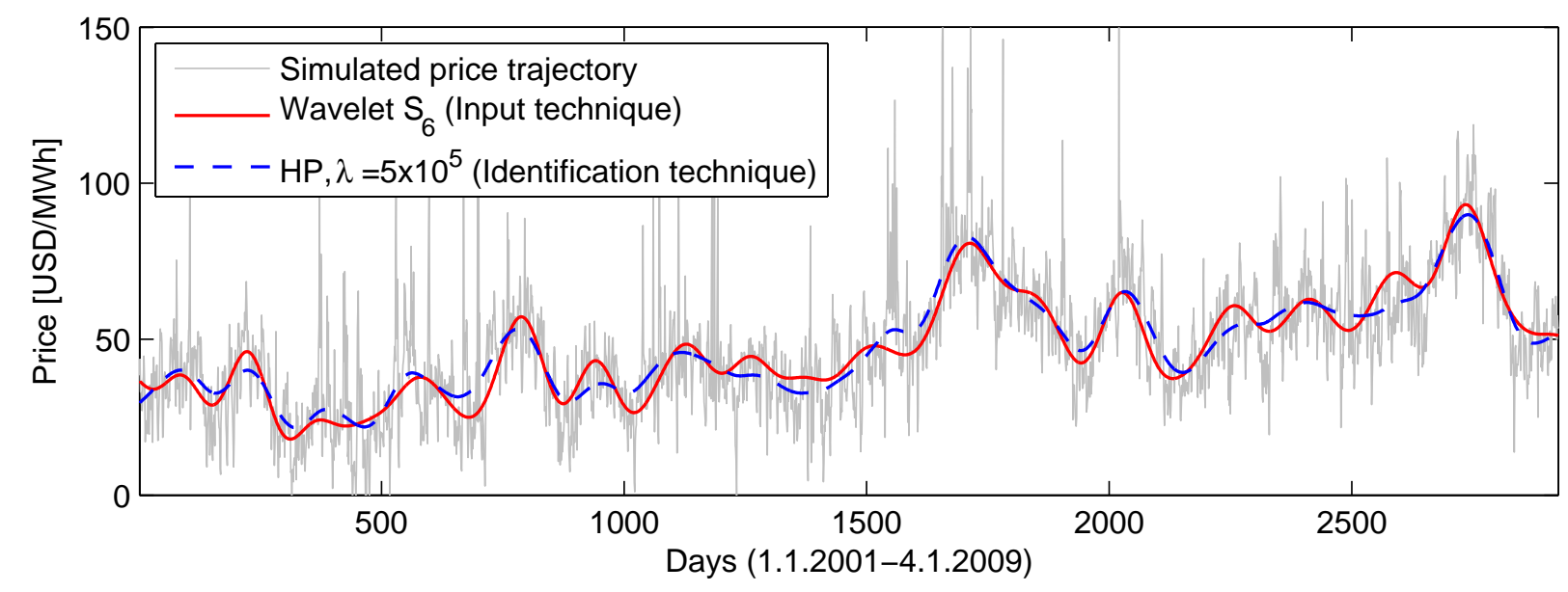

Figure 2: A sample simulated trajectory of the PJM spot prices in the 8-year period 1.1.2001-4.1.2009 together with the wavelet-based $S_{6}$ LTSC (i.e. the input technique) and the HP filter-based LTSC for $\lambda=5 \cdot 10^{5}$ (i.e. the identification technique).

Moreover, the HP filter seems to be more robust than wavelet smoothing. For instance, if we believe that the true LTSC can be well approximated by the $S_{6}$ wavelet approximation, we may either use the $S_{6}$ approximation itself or a HP filter with $\lambda \approx 5 \cdot 10^{5}$. However, if our assumption is incorrect and the true LTSC is better described by, say, the $S_{5}$ wavelet approximation, the fit of the HP filter will probably be closer to the true LTSC than the fit of the $S_{6}$ approximation, as can be seen in Table 1 . This robustness is a very important feature because in practice we do not know the true shape of the LTSC.

\section{Conclusions}

In this paper we propose a simple tool for identification of the long-term (trend-)seasonal component (LTSC) in time series of daily electricity spot prices. We argue that the filter proposed by Hodrick and Prescott (1997) for growth-cycle GDP decomposition can be successfully used in the context of electricity markets. The method is simple and allows for an accurate identification of the seasonal pattern. In terms of flexibility (i.e. ability to yield a smooth and relatively accurate fit to various spot price time series) it is comparable to the wavelet-based LTSC. In terms of computational complexity it is far superior.

In the forecasting context it shares the advantages (flexibility) but also the drawbacks (extrapolation problems, choice of parameters) of the wavelet approach. However, as Nowotarski et al. (2013) have recently shown, extrapolation problems can be easily overcome. Computing a wavelet smoother for a spot price signal extrapolated into the future with a deterministic function linearly or exponentially decaying to the median spot price in the calibration period yields more accurate forecasts of the LTSC than an extrapolation of monthly dummies or a sine-based LTSC. Exactly the same procedure can be applied for the HP filter. Moreover, having only one free parameter ( $\lambda$ ) the HP filter is easier to use and less prone to overfitting than wavelet techniques, where the number of 'parameters' is much richer and includes the decomposition level and the wavelet fam- 
Table 1: The mean squared errors (MSE) between the 'original' LTSC (Input technique) and the estimated LTSC (Identification technique). The values are expressed as percentage surplus over the minimum MSE for a particular input technique, i.e. zero means that the technique performed best, while any value $x \%$ greater than zero means that a particular technique had MSE $x \%$ higher than the best technique. All values under $15 \%$ are emphasized in bold. Note that not always the input technique turns out to be the best estimation technique.

\begin{tabular}{|c|c|c|c|c|c|c|c|c|c|c|c|c|}
\hline \multirow[b]{3}{*}{ Input } & \multicolumn{12}{|c|}{ Identification technique (estimated LTSC model) } \\
\hline & \multicolumn{7}{|c|}{ HP filter-based $(\lambda=\ldots)$} & \multicolumn{4}{|c|}{ Wavelet-based } & \multirow{2}{*}{$\begin{array}{l}\text { Sine- } \\
\text { based }\end{array}$} \\
\hline & $5 \cdot 10^{4}$ & $1 \cdot 10^{5}$ & $5 \cdot 10^{5}$ & $1 \cdot 10^{6}$ & $5 \cdot 10^{6}$ & $1 \cdot 10^{7}$ & $5 \cdot 10^{7}$ & $S_{5}$ & $S_{6}$ & $S_{7}$ & $S_{8}$ & \\
\hline & \multicolumn{12}{|c|}{ Nord Pool market (3 years: 2011-2013) } \\
\hline$S_{5}$ & $41 \%$ & $78 \%$ & $156 \%$ & $182 \%$ & $259 \%$ & $313 \%$ & $469 \%$ & $\mathbf{0 \%}$ & $201 \%$ & $237 \%$ & $655 \%$ & $269 \%$ \\
\hline$S_{6}$ & $42 \%$ & $24 \%$ & $\mathbf{0 \%}$ & $3 \%$ & $53 \%$ & $102 \%$ & $253 \%$ & $122 \%$ & $23 \%$ & $35 \%$ & $452 \%$ & $106 \%$ \\
\hline$S_{7}$ & $93 \%$ & $67 \%$ & $21 \%$ & $9 \%$ & $26 \%$ & $67 \%$ & $229 \%$ & $195 \%$ & $68 \%$ & $\mathbf{0 \%}$ & $476 \%$ & $80 \%$ \\
\hline$S_{8}$ & $272 \%$ & $229 \%$ & $143 \%$ & $113 \%$ & $55 \%$ & $35 \%$ & $\mathbf{0 \%}$ & $440 \%$ & $237 \%$ & $98 \%$ & $21 \%$ & $142 \%$ \\
\hline \multirow[t]{2}{*}{ Sine } & $51 \%$ & $35 \%$ & $7 \%$ & $\mathbf{0 \%}$ & $6 \%$ & $29 \%$ & $126 \%$ & $121 \%$ & $43 \%$ & $4 \%$ & $290 \%$ & $24 \%$ \\
\hline & \multicolumn{12}{|c|}{ EEX market (5 years: $2006-2010)$} \\
\hline$S_{5}$ & $12 \%$ & $37 \%$ & $132 \%$ & $178 \%$ & $271 \%$ & $315 \%$ & $438 \%$ & $\mathbf{0 \%}$ & $155 \%$ & $325 \%$ & $558 \%$ & $328 \%$ \\
\hline$S_{6}$ & $10 \%$ & $\mathbf{0 \%}$ & $22 \%$ & $51 \%$ & $140 \%$ & $194 \%$ & $359 \%$ & $88 \%$ & $1 \%$ & $207 \%$ & $512 \%$ & $263 \%$ \\
\hline$S_{7}$ & $96 \%$ & $66 \%$ & $14 \%$ & $\mathbf{0 \%}$ & $8 \%$ & $46 \%$ & $218 \%$ & $240 \%$ & $82 \%$ & $10 \%$ & $381 \%$ & $217 \%$ \\
\hline$S_{8}$ & $226 \%$ & $178 \%$ & $92 \%$ & $64 \%$ & $15 \%$ & $\mathbf{0 \%}$ & $2 \%$ & $443 \%$ & $195 \%$ & $63 \%$ & $4 \%$ & $248 \%$ \\
\hline \multirow[t]{2}{*}{ Sine } & $21 \%$ & $\mathbf{8 \%}$ & $\mathbf{0 \%}$ & $4 \%$ & $29 \%$ & $51 \%$ & $134 \%$ & $98 \%$ & $30 \%$ & $37 \%$ & $207 \%$ & $119 \%$ \\
\hline & \multicolumn{12}{|c|}{ PJM market (8 years: 2001-2008) } \\
\hline$S_{5}$ & 0\% & $14 \%$ & $75 \%$ & $109 \%$ & $187 \%$ & $218 \%$ & $294 \%$ & $9 \%$ & $89 \%$ & $225 \%$ & $328 \%$ & $225 \%$ \\
\hline$S_{6}$ & $15 \%$ & $2 \%$ & $8 \%$ & $32 \%$ & $114 \%$ & $152 \%$ & $249 \%$ & $93 \%$ & $0 \%$ & $162 \%$ & $305 \%$ & $196 \%$ \\
\hline$S_{7}$ & $112 \%$ & $80 \%$ & $24 \%$ & $7 \%$ & $2 \%$ & $19 \%$ & $111 \%$ & $259 \%$ & $88 \%$ & 0\% & $173 \%$ & $164 \%$ \\
\hline$S_{8}$ & $297 \%$ & $237 \%$ & $129 \%$ & $94 \%$ & $33 \%$ & $15 \%$ & $11 \%$ & $569 \%$ & $251 \%$ & $83 \%$ & 0\% & $240 \%$ \\
\hline Sine & $28 \%$ & $14 \%$ & 0\% & $1 \%$ & $18 \%$ & $31 \%$ & $80 \%$ & $105 \%$ & $32 \%$ & $35 \%$ & $89 \%$ & $91 \%$ \\
\hline
\end{tabular}

ily and order. Finally we note that we cannot point to a single, optimal value of the smoothing parameter. However, if we were to suggest one value then $\lambda=5 \cdot 10^{5}$ would be a reasonable rule of thumb. Our empirical study indicates that such a value of the smoothing parameter yields a good performance across a wide range of datasets.

\section{Acknowledgements}

This work was supported by funds from the National Science Centre (NCN, Poland) through grant no. 2011/01/B/HS4/01077 (to RW) and from the Ministry of Science and Higher Education (MNiSW, Poland) through the 'Diamond grant' scheme, grant no. DI 2011/0183/41 (to MZ).

\section{Bibliography}

Benth, F.E., Kiesel, R., Nazarova, A. (2012) A critical empirical study of three electricity spot price models. Energy Economics 34(5), 1589-1616.

Bierbrauer, M., Menn, C., Rachev, S.T., Trück, S. (2007) Spot and derivative pricing in the EEX power market. Journal of Banking and Finance 31, 3462-3485.

Bordignon, S., Bunn, D. W., Lisi, F., Nan, F. (2013), Combining day-ahead forecasts for British electricity prices. Energy Economics 35, 88-103. 
Conejo, A.J., Contreras, J., Espinola, R., Plazas, M.A. (2005) Forecasting electricity prices for a day-ahead pool-based electric energy market. International Journal of Forecasting 21(3), 435-462.

De Jong, C. (2006) The nature of power spikes: A regime-switch approach. Studies in Nonlinear Dynamics \& Econometrics 10(3), Article 3.

Fanone, E., Gamba, A., Prokopczuk, M. (2013) The case of negative day-ahead electricity prices. Energy Economics 35, 22-34.

Fleten, S.-E., Heggedal, A.M., Siddiqui, A. (2011) Transmission capacity between Norway and Germany: a real options analysis. Journal of Energy Markets 4(1), 121-147.

Gianfreda, A., Grossi, L. (2012) Forecasting Italian electricity zonal prices with exogenous variables. Energy Economics 34(6), 2228-2239.

Haugom, E., Ullrich, C.J. (2012) Forecasting spot price volatility using the short-term forward curve. Energy Economics 34, 1826-1833.

Higgs, H., Worthington, A. (2008) Stochastic price modeling of high volatility, mean-reverting, spike-prone commodities: The Australian wholesale spot electricity market. Energy Economics 30, 3172-3185.

Hodrick, R.J., Prescott, E.C. (1980) Postwar U.S. business cycles: An empirical investigation. Carnegie-Mellon University Working Paper.

Hodrick, R.J., Prescott, E.C. (1997) Postwar U.S. business cycles: An empirical investigation. Journal of Money, Credit and Banking 29(1), 1-16.

Janczura, J., Trueck, S., Weron, R., Wolff, R. (2013) Identifying spikes and seasonal components in electricity spot price data: A guide to robust modeling. Energy Economics 38, 96-110.

Janczura, J., Weron, R. (2010) An empirical comparison of alternate regime-switching models for electricity spot prices. Energy Economics 32, 1059-1073.

Janczura, J., Weron, R. (2012) Efficient estimation of Markov regime-switching models: An application to electricity spot prices. AStA - Advances in Statistical Analysis 96(3), 385-407.

Keles, D., Hartel, R., Möst, D., Fichtner, W. (2012) Compressed-air energy storage power plant investments under uncertain electricity prices: An evaluation of compressed-air energy storage plants in liberalized energy markets. Journal of Energy Markets 5(1), 53-84.

Nowotarski, J., Tomczyk, J., Weron, R. (2013) Robust estimation and forecasting of the long-term seasonal component of electricity spot prices. Energy Economics 39, 13-27.

Schlueter, S. (2010) A long-term/short-term model for daily electricity prices with dynamic volatility, Energy Economics 32, 1074-1081.

Stevenson, M.J., Amaral, J.F.M., Peat, M. (2006) Risk management and the role of spot price predictions in the Australian retail electricity market. Studies in Nonlinear Dynamics and Econometrics 10(3), Article 4.

Trück, S., Weron, R., Wolff, R. (2007) Outlier treatment and robust approaches for modeling electricity spot prices. Proceedings of the 56th Session of the ISI. Available at MPRA: http://mpra.ub.uni-muenchen.de/4711/.

Weron, R. (2006) Modeling and Forecasting Electricity Loads and Prices: A Statistical Approach. Wiley, Chichester.

Weron, R. (2008) Market price of risk implied by Asian-style electricity options and futures. Energy Economics 30, 1098-1115.

Weron, R. (2014) A review of electricity price forecasting: The past, the present and the future, submitted. Working paper version available from RePEc: http://ideas.repec.org/p/wuu/wpaper/hsc1402.html. 


\section{HSC Research Report Series 2014}

For a complete list please visit http://ideas.repec.org/s/wuu/wpaper.html

01 Diffusion and adoption of dynamic electricity tariffs: An agent-based modeling approach by Anna Kowalska-Pyzalska, Katarzyna Maciejowska, Katarzyna Sznajd-Weron and Rafał Weron

02 A review of electricity price forecasting: The past, the present and the future by Rafał Weron

03 Merging quantile regression with forecast averaging to obtain more accurate interval forecasts of Nord Pool spot prices by Jakub Nowotarski and Rafał Weron

04 A note on using the Hodrick-Prescott filter in electricity markets by Rafał Weron and Michał Zator 